\title{
A Robust Color Image Watermarking Scheme Using Entropy and QR Decomposition
}

\author{
Lauri LAUR ${ }^{1}$, Pejman RASTI ${ }^{3}$, Mary AGOYI ${ }^{2}$, Gholamreza ANBARJAFARI ${ }^{3,4}$ \\ ${ }^{1}$ Institute of Computer Science, University of Tartu, Liivi 2, 50409, Tartu, Estonia \\ ${ }^{2}$ Faculty of Engineering, Cyprus International University, Lefkoşa, KKTC, via Mersin 10, Turkey \\ ${ }^{3}$ iCV Research Group, Institute of Technology, University of Tartu, Nooruse 1,Tartu 50411, Estonia \\ ${ }^{4}$ Dept. of Electrical and Electronic Eng., Hasan Kalyoncu University, Gaziantep, Turkey \\ lauri01@ut.ee, pejman.rasti@ut.ee,magoyi@ciu.edu.tr,shb@ut.ee
}

\begin{abstract}
Internet has affected our everyday life drastically. Expansive volumes of information are exchanged over the Internet consistently which causes numerous security concerns. Issues like content identification, document and image security, audience measurement, ownership, copyrights and others can be settled by using digital watermarking. In this work, robust and imperceptible nonblind color image watermarking algorithm is proposed, which benefit from the fact that watermark can be hidden in different color channel which results into further robustness of the proposed technique to attacks. Given method uses some algorithms such as entropy, discrete wavelet transform, Chirp z-transform, orthogonal-triangular decomposition and Singular value decomposition in order to embed the watermark in a color image. Many experiments are performed using well-known signal processing attacks such as histogram equalization, adding noise and compression. Experimental results show that the proposed scheme is imperceptible and robust against common signal processing attacks.
\end{abstract}

\section{Keywords}

Chirp Z Transform, Color Image Watermarking, Discrete Wavelet Transform, Entropy, Orthogonal-triangular decomposition, QR decomposition, Singular Value Decomposition

\section{Introduction}

With popularization on the World Wide Web and ease of data transferring over the Internet, copyright and security concerns have emerged. As computational performance has become cheaper, it is nowadays cheaper to copy and distribute digital media than make one. In order to protect digital media from piracy, unauthorized use and other illegal actions, watermarks are used. Watermarking is a method to embed data into host signal by slightly modifying its content [1]. Watermarking algorithms can be blind, semi-blind and non-blind. Method is blind when original objects are not used to extract the watermark from watermarked signal, only secret key is required [2]. Semi-blind watermarking techniques need secret key and original watermark to extract embedded watermark from watermarked signal [3]. Non-blind watermarking technique requires original signal, watermark sequence and secret key to extract watermark from watermarked image [4]. Watermarking scheme has to be able to survive common signal processing attacks like JPEG compression, Gaussian noise, blurring, contrast enhancement and others [5].

Such geometrical attacks are not trying to remove the watermark, instead they try to distort the watermark detection system [6]. Robustness can also be a degree of which watermarking scheme can resist modifications of a host signal [7]. Other important quality of watermarking algorithm is imperceptibility. Watermark imperceptibility expresses similarity between original and watermarked image [8]. To evaluate the perceptual quality of watermarked image Peak-Signal-to-Noise-Ratio (PSNR) can be used [9]. It is widely used metric in image processing, what uses mean square error calculation to assess objectively visual quality [10]. Robustness and imperceptibility confine each other. Good algorithms have to make compromises to preserve robustness and make watermark inside watermarked image imperceptible [11].

Correlation Coefficient (CC) metric can be used to evaluate robustness of watermarking technique. It is used to measure linear association [12]. There are many alternatives, like sum of absolute difference and sum of squared differences, to correlation coefficient, but they are not invariant to contrast and brightness variations, therefore correlation coefficient is more robust [13]. The higher the correlation coefficient absolute value, the stronger the relationship is between the original and the extracted watermark [14].

Digital watermarking algorithms can generally be divided into two categories, spatial domain and frequency domain methods using transformations such as Discrete Cosine Transform (DCT), Discrete Sine Transform (DST), Discrete Hartley Transform (DHT) and Discrete Wavelet Transform (DWT) [15-17].

DWT is a commonly used technique in signal processing and it is also used in image compression standard JPEG2000 [18]. DWT produces time-scale representation 
of the digital image using digital filtering methods. Onelevel two-dimensional DWT generates four bands of data. Watermark is usually embedded into low-pass band, because it is more robust against attacks [19]. Entropy is used in watermarking to measure complexity and uncertainty in signal. Entropy value is greater in an image where complexity is larger. Repeated signal processing attacks change image parts where entropy value is higher [20]. QR decomposition, also known as orthogonal-triangular decomposition, is factorizing matrix to unitary matrix and uppertriangular matrix. It is beneficial in watermarking because it enhances the robustness and security of the image [21]. Chirp Z-Transform (CZT) is a method for computing samples of z-transform. CZT helps to get robust and intensely imperceptible watermarked image [22]. Singular Value Decomposition (SVD) is a mathematical technique for extracting algebraic properties from an image. Singular values gotten from SVD have good stability, smaller changes on an image do not affect singular values very much [23].

Image watermarking is rapidly emerging field and many good algorithms have been proposed. One of the simplest methods is Least Significant Bit (LSB) algorithm. In spatial domain, an image is presented by pixel values. LSB method embeds watermark into image's pixels by modifying least significant bits of pixel values. LSB is computationally very efficient and it also gives quite good perceptual results. On the other hand, it is not robust against common signal processing attacks and after most attacks it is not possible to recover the watermark [24]. Lai et al. proposed a hybrid watermarking algorithm [25] that uses DWT and SVD. They embed a watermark into singular values of intermediate frequency subbands. They claim that their approach needs less SVD computation resources and it preserves better visual perception of images than most existing DWT-SVD based algorithms. Agoyi et al. proposed a novel watermarking scheme [22] based on DWT, CZT and SVD. They also embedded a watermark into singular values. Several experiments were conducted and it was found that their algorithm is imperceptible and robust in various signal processing operations. L. Ghouti and A. M. Andalusi proposed colored image watermarking technique [26] that uses Spatio-Chromatic Fourier Transform with spread-spectrum signaling combined with error correction using semi-random low density parity check codes. They found that their algorithm is robust against standard signal processing attacks like additive white Gaussian noise and JPEG compression.

In this study we have proposed a novel non-blind color image watermarking scheme using DWT, CZT, QR decomposition, SVD and entropy. Watermark is embedded into low entropy parts of all three RGB color channels. The developed technique shows good robustness characteristics and satisfies imperceptibility requirement.

The remainder of this paper is organized as follows. In Sec. 2 there is a detailed overview of the proposed approach. Section 3 contains the outcome of experimental results and in Sec. 4 we conclude the paper.

\section{Proposed Approach}

The proposed algorithm embeds watermark into color image's three color channels, red, green and blue (RGB). It uses entropy to find image blocks with low complexity and embeds watermark by combining best characteristics of DWT, CZT, QR decomposition and SVD. More detailed description with formulas and flowcharts of the proposed embedding and extracting algorithm will be provided in this section.

In order to embed the watermark, an original color image is divided into three color channels, red, green and blue. Each color channel of the input image is then divided into four equal sized blocks and entropy of each block is found and the average entropy value is taken as the threshold. The watermark is embedded into each block of every color channel that has entropy value lower than the calculated threshold. To embed the watermark into color channels block, each block is decomposed using one-level two dimensional DWT to obtain four frequency subbands. CZT is then applied to low-frequency subband from DWT and QR decomposition is applied to the result, then diagonal matrix is calculated from it. SVD is applied to calculated diagonal matrix to obtain block's singular values. SVD is also applied to watermark image and singular values from there are added together with block's singular values. After that, inverse SVD, QR decomposition, CZT and DWT are used to get watermarked block. Modified blocks are added together with higher entropy blocks and watermarked color channel is obtained. Lastly all three color channels are put together to get back watermarked color image.

Watermark extraction is also done for all three color channels separately. Original image's color channel is divided into blocks and entropy of each block is found. The following is done to all original image's color channel blocks that have entropy value lower than the average entropy and for corresponding watermarked image's color channel blocks. Both image's blocks are then decomposed using one-level two dimensional DWT. CZT is applied to both calculated low-frequency subbands to transform it to Z-domain. QR decomposition is applied to both results of the previous step and diagonal matrices are calculated. SVD is applied to both diagonal matrices and also to watermark image. Original image's singular values are subtracted from watermarked image's singular values to get extracted watermark singular values. Using extracted singular values and unitary matrices got from watermark image, inverse SVD is used to get extracted watermark image. More detailed mathematical description about embedding and extracting algorithm can be found in the following subsections.

\subsection{Watermark Embedding}

Watermark embedding scheme is presented in Fig. 1 and explained in the following.

Divide the original colored cover image into three color channels R, G and B. Apply the following steps to 


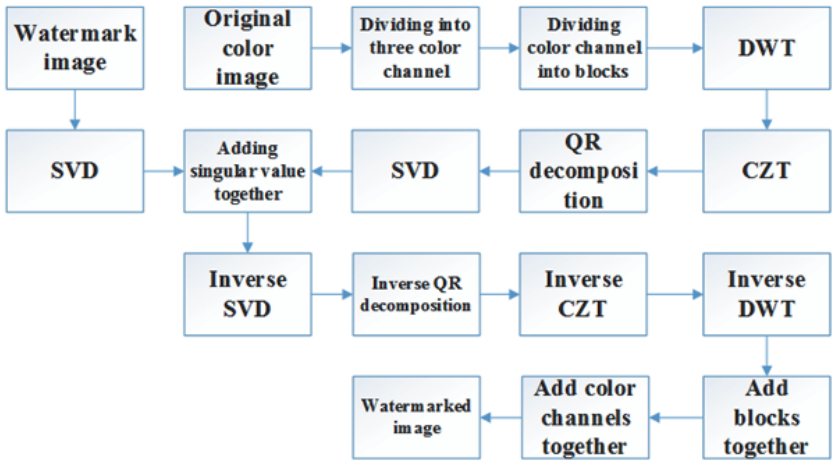

Fig. 1. Block diagram of embedding scheme.

each channel separately.

Divide $m \times n$ color channel into $\alpha \times \beta$ blocks, where $\alpha$ divides $m$ and $\beta$ divides $n$. Let $M=m / \alpha$ and $N=n / \beta$. Then each block can be described as in (1)

$$
\mathbf{B}_{m n} m \in\{1 \ldots M\}, n \in\{1 \ldots N\} \text {. }
$$

Calculate entropy value for each block, where the entropy value is designated as $E$.

Calculate the average of all entropy values $E$ from all blocks and denote the outcome as the threshold $T$. $T$ can be calculated as given in (2)

$$
T=\sum_{m=1}^{M} \sum_{n=1}^{N} \frac{E\left(\mathbf{B}_{m n}\right)}{M \cdot N} .
$$

Use one-level DWT on each block with entropy value $E$ less than the calculated threshold $T$ to decompose it into four subbands as given in (3)

$$
\begin{aligned}
& \mathbf{L L}_{m n} \mathbf{L H}_{m n} \mathbf{H} \mathbf{L}_{m n} \mathbf{H} \mathbf{H}_{m n}=\operatorname{DWT}\left(\mathbf{B}_{m n}\right), \\
& \forall \mathbf{B}_{m n} \in\left\{\mathbf{B}_{m n}: E\left(\mathbf{B}_{m n}\right)<T\right\}
\end{aligned}
$$

Calculate CZT of low-frequency subband $\mathbf{L} \mathbf{L}_{m n}$ for all decomposed blocks as given in (4)

$$
\mathbf{C}_{m n}=C Z T\left(\mathbf{L} \mathbf{L}_{m n}\right) \text {. }
$$

Apply QR decomposition to matrix $\mathbf{C}_{m n}$ from (4) to calculate diagonal matrix as given in (5)

$$
\begin{gathered}
{\left[\mathbf{Q}_{m n} \mathbf{R}_{m n}\right]=Q R\left(\mathbf{C}_{m n}\right),} \\
\mathbf{D} 1_{m}=\operatorname{diag}\left(\mathbf{R}_{m n}\right), \\
\mathbf{D}_{m n}=\operatorname{zeros}\left(\mathbf{R}_{m n}\right), \\
\mathbf{D}_{m m}=\mathbf{D} 1_{m} .
\end{gathered}
$$

Apply SVD to diagonal matrix $\mathbf{D}_{m n}$ from (5) to further decompose it as shown in (6)

$$
\left[\mathbf{U}_{m n} \mathbf{S}_{m n} \mathbf{V}_{m n}\right]=\operatorname{SVD}\left(\mathbf{D}_{m n}\right)
$$

Apply SVD to watermark image $\boldsymbol{W}$ and decompose it as shown in (7)

$$
\left[\mathbf{U}_{1} \mathbf{S}_{1} \mathbf{V}_{1}\right]=S V D(\mathbf{W})
$$

Calculate new singular values by adding original image's decomposed singular values to watermark image's singular values multiplied by scaling factor $\gamma \cdot \gamma$ is for controlling the strength of the added watermark. This is shown in (8)

$$
\mathbf{S}_{2 m n}=\mathbf{S}_{m n}+\gamma \mathbf{S}_{1}
$$

Combine unitary matrices $\mathbf{U}_{m n}$ and $\mathbf{V}_{m n}$ from the decomposed original image with new singular values calculated in (8) as shown in (9)

$$
\mathbf{D}_{2 m n}=\mathbf{U}_{m n} \cdot \mathbf{S}_{2 m n} \cdot \mathbf{V}_{m n}^{T} .
$$

Replace upper-triangular matrix $\mathbf{R}_{m n}$ diagonal values with modified diagonal matrix $\mathbf{D}_{2 m n}$ as shown in (10)

$$
\mathbf{R}_{m m}=\mathbf{D}_{2 m m} .
$$

Combine unitary matrix $\mathbf{Q}_{m n}$ with modified uppertriangular matrix $\mathbf{R}_{m n}$ as shown in (11)

$$
\mathbf{C}_{2 m n}=\mathbf{Q}_{m n} \cdot \mathbf{R}_{m n} .
$$

Calculate inverse CZT of $\mathbf{C}_{2 m n}$ to get watermarked low-frequency subband as shown in (12)

$$
\mathbf{L L}_{2 m n}=\operatorname{ICZT}\left(\mathbf{C}_{2 m n}\right) \text {. }
$$

Calculate inverse DWT to get watermarked image block. Instead of $\mathbf{L} \mathbf{L}_{m n}$ use modified $\mathbf{L} \mathbf{L}_{2 m n}$ as shown in (13)

$$
\mathbf{I}_{m n}=I D W T\left(\mathbf{L L}_{2 m n} \mathbf{L H}_{m n} \mathbf{H L}_{m n} \mathbf{H} \mathbf{H}_{m n}\right) .
$$

Add together modified low entropy blocks with high entropy blocks and all three color channels to get the watermarked color image.

\subsection{Watermark Extraction}

Watermark extraction scheme is presented in Fig. 2 and explained in the following.

Divide original colored cover image into three color channels R, G and B. Apply the following steps to each channel separately.

Divide $m \times n$ original image color channel into $\alpha \times \beta$ blocks, where $\alpha$ divides $m$ and $\beta$ divides $n$. Let $M=m / \alpha$ and $N=n / \beta$. Then each block can be described as in (14)

$$
\mathbf{B}_{m n} \quad m \in\{1 \ldots M\}, n \in\{1 \ldots N\} .
$$

Divide watermarked image corresponding color channel similarly into $\alpha \times \beta$ blocks, where each block can be described as in (15)

$$
\mathbf{B}^{\prime}{ }_{m n} \quad m \in\{1 \ldots M\}, n \in\{1 \ldots N\} .
$$

Calculate entropy value for each block of the original image's color channel, where the entropy value is designated as $E$.

Calculate the average of all entropy values $E$ for all blocks of the original image's color channel and denote the outcome as the threshold $T$. $T$ can be calculated as in (16) 


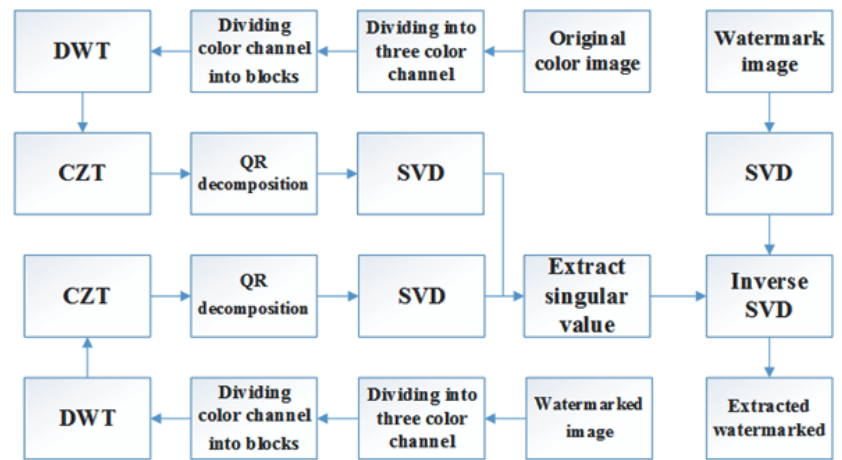

Fig. 2. Block diagram of extracting scheme.

$$
T=\sum_{m=1}^{M} \sum_{n=1}^{N} \frac{E\left(\mathbf{B}_{m n}\right)}{M \cdot N} .
$$

Use one-level DWT on each original image block with entropy value $E$ less than the calculated threshold $T$ to decompose it into four subbands as given in (17)

$$
\begin{aligned}
& \mathbf{L L}_{m n} \mathbf{L H}_{m n} \mathbf{H} \mathbf{L}_{m n} \mathbf{H} \mathbf{H}_{m n}=\operatorname{DWT}\left(\mathbf{B}_{m n}\right), \\
& \forall \mathbf{B}_{m n} \in\left\{\mathbf{B}_{m n}: E\left(\mathbf{B}_{m n}\right)<T\right\} .
\end{aligned}
$$

Use one-level DWT on corresponding watermarked image block to decompose it into four subbands as given in (18)

$$
\begin{aligned}
& \mathbf{L L}_{m n}^{\prime} \mathbf{L} \mathbf{H}_{m n}^{\prime} \mathbf{H L}_{m n}^{\prime} \mathbf{H H}_{m n}=\operatorname{DWT}\left(\mathbf{B}_{m n}^{\prime}\right), \\
& \forall \mathbf{B}_{m n}^{\prime} \in\left\{\mathbf{B}_{m n}^{\prime}: E\left(\mathbf{B}_{m n}\right)<T\right\} .
\end{aligned}
$$

Calculate CZT of low-frequency subband $\mathbf{L} \mathbf{L}_{m n}$ for all decomposed blocks as given in (19)

$$
\mathbf{C}_{m n}=C Z T\left(\mathbf{L} \mathbf{L}_{m n}\right) \text {. }
$$

Calculate CZT of low-frequency subband $\mathbf{L L}{ }_{m n}$ for all decomposed blocks as given in (20)

$$
\mathbf{C}_{m n}^{\prime}=\operatorname{CZT}\left(\mathbf{L} \mathbf{L}_{m n}^{\prime}\right) \text {. }
$$

Apply QR decomposition to matrix $\mathbf{C}_{m n}$ from (19) to calculate diagonal matrix as given in (21)

$$
\begin{gathered}
{\left[\mathbf{Q}_{m n} \mathbf{R}_{m n}\right]=Q R\left(\mathbf{C}_{m n}\right),} \\
\mathbf{D} 1_{m}=\operatorname{diag}\left(\mathbf{R}_{m n}\right), \\
\mathbf{D}_{m n}=\operatorname{zeros}\left(\mathbf{R}_{m n}\right), \\
\mathbf{D}_{m m}=\mathbf{D} 1_{m} .
\end{gathered}
$$

Apply QR decomposition to matrix $\mathbf{C}^{\prime}{ }_{m n}$ from (20) to calculate diagonal matrix as given in (22)

$$
\begin{gathered}
{\left[\mathbf{Q}_{m n}^{\prime} \mathbf{R}_{m n}^{\prime}\right]=Q R\left(\mathbf{C}_{m n}^{\prime}\right),} \\
\mathbf{D} 1_{m}^{\prime}=\operatorname{diag}\left(\mathbf{R}_{m n}^{\prime}\right), \\
\mathbf{D}_{m n}^{\prime}=\operatorname{zeros}\left(\mathbf{R}_{m n}^{\prime}\right), \\
\mathbf{D}_{m m}^{\prime}=\mathbf{D} 1_{m}^{\prime} .
\end{gathered}
$$

Apply SVD to diagonal matrix $\mathbf{D}_{m n}$ from (21) to further decompose it as shown in (23)

$$
\left[\mathbf{U}_{m n} \mathbf{S}_{m n} \mathbf{V}_{m n}\right]=S V D\left(\mathbf{D}_{m n}\right)
$$

Apply SVD to diagonal matrix $\mathbf{D}_{m n}$ from (22) to further decompose it as shown in (24)

$$
\left[\mathbf{U}_{m n}^{\prime} \mathbf{S}_{m n}^{\prime} \mathbf{V}_{m n}^{\prime}\right]=\operatorname{SVD}\left(\mathbf{D}_{m n}^{\prime}\right) .
$$

Apply SVD to watermark image $\mathbf{W}$ and decompose it as shown in (25)

$$
\left[\mathbf{U}_{1} \mathbf{S}_{1} \mathbf{V}_{1}\right]=S V D(\mathbf{W})
$$

Subtract singular values of original image's block from singular values of watermarked image's block and divide the outcome by scaling factor $\gamma$ to get singular values of extracted watermark image as shown in (26)

$$
\mathbf{S}_{1 m n}^{\prime}=\left(\mathbf{S}_{m n}^{\prime}-\mathbf{S}_{m n}\right) / \gamma \text {. }
$$

Combine unitary matrices $\mathbf{U}_{1}$ and $\mathbf{V}_{1}$ from the watermarked image with extracted singular values calculated in (26) to get extracted watermark for each block as shown in (27)

$$
\mathbf{W I}_{m n}=\mathbf{U}_{1} \cdot \mathbf{S}_{1 m n}^{\prime} \cdot \mathbf{V}_{1}^{T} \text {. }
$$

Change the extracted watermark image into black and white image by using average of the image as threshold.

\section{Experimental Results}

Various experiments were conducted during this research, numerous host images were watermarked with different watermark images. Cover images that were used in experiments were color images including well known benchmarks like "Lena", "Barbara" and "Rose" and images from an uncompressed color image database [1]. Watermark images were $128 \times 128$ grayscale images. Figure 3 shows watermark images "Cameraman", "Signature" and "UT Logo" used for experimental results.

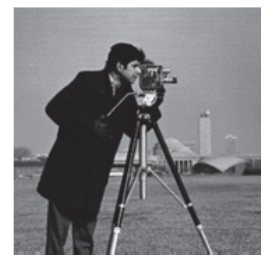

(a) Cameraman

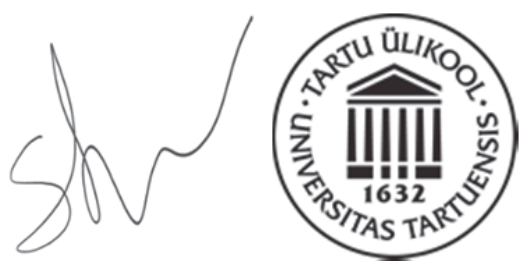

(c) UT logo
Fig. 3. (a), (b) and (c) are the watermark images.

Figure 4 presents (a) the host image "Lena", (b) watermarked host image and (c - n) watermarked images with different attacks applied to it.

In order to evaluate imperceptibility characteristics quality measurement PSNR was used. It measures image quality in decibels and image with over $40 \mathrm{~dB}$ is considered to have high quality [2]. PSNR values of the proposed method is compared with Lai \& Tsai method [3], LSB method [4] and Agoyi et al. proposed method [5], results of this comparison are shown Tab. 1. 


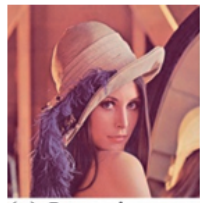

(b) Lena watermar

with Cameraman

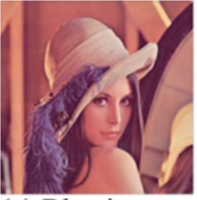

(e) Blurring attack

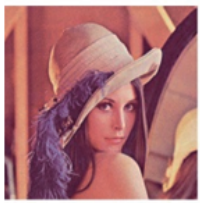

(i) Gaussian

noise

attack

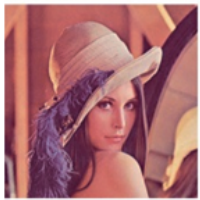

(m) Scaling attack
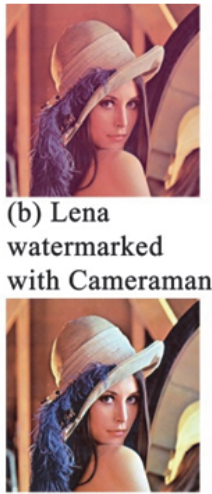

(f) Contrast

enhancement attack

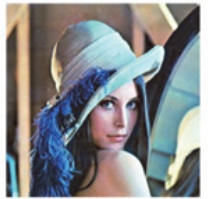

(j) Histogram equalization attack (a) Lena image the original image watermarked with "Cameraman" image, (c-n) are watermarked images with different attacks.

\begin{tabular}{|c|c|c|c|}
\hline Method & $\begin{array}{c}\text { Watermark } \\
\text { image }\end{array}$ & Lena & $\begin{array}{c}\text { ucid00136 } \\
{[16]}\end{array}$ \\
\hline \multirow{3}{*}{ LSB } & Signature & 50.87 & 51.23 \\
\hline & Cameraman & 50.88 & 51.12 \\
\hline & UT logo & 50.65 & 51.06 \\
\hline \multirow{3}{*}{$\begin{array}{l}\text { Lai \& Tsai } \\
\text { method }\end{array}$} & Signature & 26.36 & 25.21 \\
\hline & Cameraman & 28.14 & 27.10 \\
\hline & UT logo & 33.02 & 34.44 \\
\hline \multirow{3}{*}{$\begin{array}{l}\text { Agoyi et al. } \\
\text { proposed } \\
\text { method [22] }\end{array}$} & Signature & 33.89 & 35.50 \\
\hline & Cameraman & 38.08 & 39.77 \\
\hline & UT logo & 47.37 & 48.54 \\
\hline \multirow{3}{*}{$\begin{array}{l}\text { Proposed } \\
\text { method }\end{array}$} & Signature & 85.21 & 85.42 \\
\hline & Cameraman & 86.78 & 87.01 \\
\hline & UT logo & 91.66 & 92.17 \\
\hline
\end{tabular}

Tab. 1. PSNR (dB) values images after embedding the watermark by using different algorithms.

To evaluate robustness properties of our proposed method various attacks like additive white Gaussian noise, blurring, contrast enhancement, cropping, flipping, gamma correction, Gaussian noise, histogram equalization, JPEG compression, salt and pepper noise, scaling and sharpening were used on watermarked image. The extracted watermark image was evaluated using correlation coefficient metric. It evaluates similarity between the extracted watermark and the original watermark image. For comparison purposes one conventional (LSB) and two state-of-the-art (Lai \& Tsai [3] and Agoyi et al. [5]) techniques were implemented and measured to evaluate the proposed method's experi- mental results. From conducted tests we can conclude that our presented watermarking scheme outperforms other novel methods. Experimental results show that the proposed algorithm performs extremely well when histogram equalization, blurring, contrast enhancement, sharpening and scaling attacks are applied on the watermarked image.

Table 2 shows comparison between one conventional and two state-of-the-art algorithm when "Lena" is used as host image and "Cameraman" as watermark image. Table 2 points out that the proposed algorithm has significantly better correlation coefficient results with histogram equalization, cropping at least $20 \%$ of the watermarked image, blurring, contrast enhancement, Gaussian noise, sharpening, scaling and additive white Gaussian noise attacks. Correlation coefficient result is slightly better with gamma correction attack. The proposed algorithm shows slightly worse correlation coefficient results than other compared methods with flipping, JPEG compression by factor of 2 and salt and pepper noise attacks

\begin{tabular}{|l|c|c|c|c|}
\hline \multicolumn{1}{|c|}{ Attack } & LSB & $\begin{array}{c}\text { Lai \&Tsai } \\
\text { method }\end{array}$ & $\begin{array}{c}\text { Agoyi et al. } \\
\text { proposed } \\
\text { method [22] }\end{array}$ & $\begin{array}{c}\text { Proposed } \\
\text { method }\end{array}$ \\
\hline Flipping & 0.3441 & 1 & 1 & 0.9898 \\
\hline $\begin{array}{l}\text { Histogram } \\
\text { equalization }\end{array}$ & 0.0486 & 0.8869 & 0.8713 & 0.9995 \\
\hline Cropping & 0.6417 & 0.8426 & 0.8893 & 0.9348 \\
\hline JPEG & 0.0069 & 0.8968 & 0.8837 & 0.8707 \\
\hline Blurring & 0.1412 & 0.6993 & 0.6327 & 0.9858 \\
\hline $\begin{array}{l}\text { Contrast } \\
\text { enhancement }\end{array}$ & 0.0653 & 0.9039 & 0.8943 & 0.9998 \\
\hline $\begin{array}{l}\text { Salt and } \\
\text { pepper noise }\end{array}$ & 0.9871 & 0.8947 & 0.5932 & 0.9571 \\
\hline $\begin{array}{l}\text { Gaussian } \\
\text { noise }\end{array}$ & 0.0015 & 0.833 & 0.5237 & 0.9252 \\
\hline Sharpening & 0.0745 & 0.9307 & 0.8697 & 0.9988 \\
\hline $\begin{array}{l}\text { Gamma } \\
\text { correction }\end{array}$ & 0.1077 & 0.9302 & 0.7609 & 0.9354 \\
\hline Scaling & 0.1831 & 0.7324 & 0.6313 & 0.9385 \\
\hline AWGN & 0.0015 & 0.7940 & 0.4932 & 0.8728 \\
\hline
\end{tabular}

Tab. 2. Absolute value of CC of "Lena" as host image watermarked with "Cameraman".

Table 3 shows comparison when host image "Lena" is watermarked with "Signature". Table 3 shows that the proposed method has significantly higher correlation coefficient results when histogram equalization, blurring, Gaussian noise, scaling and additive white Gaussian noise attacks are applied on the watermarked image. The proposed algorithm has slightly better results when sharpening and gamma correction attacks are performed on the watermarked image. The proposed method shows worse correlation coefficient results after flipping, cropping, JPEG compression, contrast enhancement and salt and pepper noise attacks. Due to the fact that signature is a thin line, the retrieved image will contain number of discontinuity, which directly effect on $\mathrm{CC}$ value. However this discontinuity will not effect of CC values for watermark images such as cameraman or UT Logo.

Table 4 presents comparison results when "Lena" is used as the cover image and "UT logo" as the watermark image. The table shows that the proposed algorithm performs significantly better when blurring, scaling and addi- 


\begin{tabular}{|l|c|c|c|c|}
\hline \multicolumn{1}{|c|}{ Attack } & LSB & $\begin{array}{c}\text { Lai \& } \\
\text { Tsai } \\
\text { method }\end{array}$ & $\begin{array}{c}\text { Agoyi et al. } \\
\text { proposed } \\
\text { method [22] }\end{array}$ & $\begin{array}{c}\text { Proposed } \\
\text { method }\end{array}$ \\
\hline Flipping & 0.0503 & 1 & 1 & 0.5043 \\
\hline $\begin{array}{l}\text { Histogram } \\
\text { equalization }\end{array}$ & 0.0541 & 0.3558 & 0.5659 & 0.662 \\
\hline Cropping & 0.1319 & 0.2406 & 0.5464 & 0.3925 \\
\hline JPEG & 0.0686 & 0.2856 & 0.598 & 0.4035 \\
\hline Blurring & 0.0082 & 0.2885 & 0.4435 & 0.5377 \\
\hline $\begin{array}{l}\text { Contrast } \\
\text { enhancement }\end{array}$ & 0.0126 & 0.2902 & 0.6963 & 0.6388 \\
\hline $\begin{array}{l}\text { Salt and } \\
\text { pepper noise }\end{array}$ & 0.927 & 0.3167 & 0.2717 & 0.4325 \\
\hline $\begin{array}{l}\text { Gaussian } \\
\text { noise }\end{array}$ & 0.0161 & 0.3165 & 0.265 & 0.3972 \\
\hline Sharpening & 0.01 & 0.4717 & 0.6306 & 0.6668 \\
\hline $\begin{array}{l}\text { Gamma } \\
\text { correction }\end{array}$ & 0.0415 & 0.3032 & 0.4027 & 0.4299 \\
\hline Scaling & 0.0015 & 0.4148 & 0.437 & 0.4903 \\
\hline AWGN & 0.0104 & 0.3398 & 0.2758 & 0.3878 \\
\hline
\end{tabular}

\begin{tabular}{|l|c|c|c|c|}
\hline \multicolumn{1}{|c|}{ Attack } & LSB & $\begin{array}{c}\text { Lai \&Tsai } \\
\text { method }\end{array}$ & $\begin{array}{c}\text { Agoyi et al. } \\
\text { proposed } \\
\text { method [22] }\end{array}$ & $\begin{array}{c}\text { Proposed } \\
\text { method }\end{array}$ \\
\hline Flipping & 0.1341 & 1 & 1 & 0.9556 \\
\hline $\begin{array}{l}\text { Histogram } \\
\text { equalization }\end{array}$ & 0.0073 & 0.9755 & 0.9798 & 0.9998 \\
\hline Cropping & 0.527 & 0.9214 & 0.912 & 0.9169 \\
\hline JPEG & 0.0139 & 0.9892 & 0.6395 & 0.8464 \\
\hline Blurring & 0.0072 & 0.6654 & 0.9461 & 0.9543 \\
\hline $\begin{array}{l}\text { Contrast } \\
\text { enhancement }\end{array}$ & 0.007 & 0.9797 & 0.9808 & 0.9903 \\
\hline $\begin{array}{l}\text { Salt and } \\
\text { pepper noise }\end{array}$ & 0.9122 & 0.9537 & 0.8641 & 0.9561 \\
\hline $\begin{array}{l}\text { Gaussian } \\
\text { noise }\end{array}$ & 0.0182 & 0.9184 & 0.8247 & 0.9293 \\
\hline Sharpening & 0.0011 & 0.9681 & 0.9707 & 0.9929 \\
\hline $\begin{array}{l}\text { Gamma } \\
\text { correction }\end{array}$ & 0.046 & 0.9961 & 0.8069 & 0.8495 \\
\hline Scaling & 0.0326 & 0.7375 & 0.9503 & 0.9332 \\
\hline AWGN & 0.0068 & 0.8926 & 0.8656 & 0.9337 \\
\hline
\end{tabular}

Tab. 3. Absolute value of CC of "Lena" as host image watermarked with "Signature".

tive white Gaussian noise attacks are used. Our method performs slightly better with histogram equalization, contrast enhancement, salt and pepper noise, Gaussian noise and sharpening attacks. The presented scheme has slightly worse result with cropping attack and worse outcome when flipping, JPEG compression and gamma correction attacks are applied.

Table 5 illustrates experimental results when "ucid00136" image from an uncompressed color image database [1] is used as host image and "UT logo" as watermark. Table 5 shows that the proposed method has significantly better results when cropping, blurring and scaling attacks are used. It performs slightly better with histogram equalization, contrast enhancement and sharpening attacks. Our method has slightly worse results with flipping, salt and pepper noise, Gaussian noise and additive white Gaussian noise attacks and it shows worse results when JPEG compression and gamma correction attacks are applied to the watermarked image.

\begin{tabular}{|l|c|c|c|c|}
\hline \multicolumn{1}{|c|}{ Attack } & LSB & $\begin{array}{c}\text { Lai \& Tsai } \\
\text { method }\end{array}$ & $\begin{array}{c}\text { Agoyi et al. } \\
\text { proposed } \\
\text { method [22] }\end{array}$ & $\begin{array}{c}\text { Proposed } \\
\text { method }\end{array}$ \\
\hline Flipping & 0.1341 & 1 & 1 & 0.9556 \\
\hline $\begin{array}{l}\text { Histogram } \\
\text { equalization }\end{array}$ & 0.0073 & 0.9755 & 0.9798 & 0.9998 \\
\hline Cropping & 0.527 & 0.9214 & 0.912 & 0.9169 \\
\hline JPEG & 0.0139 & 0.9892 & 0.6395 & 0.8464 \\
\hline Blurring & 0.0072 & 0.6654 & 0.9461 & 0.9543 \\
\hline $\begin{array}{l}\text { Contrast } \\
\text { enhancement }\end{array}$ & 0.007 & 0.9797 & 0.9808 & 0.9903 \\
\hline $\begin{array}{l}\text { Salt and } \\
\text { pepper noise }\end{array}$ & 0.9122 & 0.9537 & 0.8641 & 0.9561 \\
\hline $\begin{array}{l}\text { Gaussian } \\
\text { noise }\end{array}$ & 0.0182 & 0.9184 & 0.8247 & 0.9293 \\
\hline Sharpening & 0.0011 & 0.9681 & 0.9707 & 0.9929 \\
\hline $\begin{array}{l}\text { Gamma } \\
\text { correction }\end{array}$ & 0.046 & 0.9961 & 0.8069 & 0.8495 \\
\hline Scaling & 0.0326 & 0.7375 & 0.9503 & 0.9332 \\
\hline AWGN & 0.0068 & 0.8926 & 0.8656 & 0.9337 \\
\hline
\end{tabular}

Tab. 4. Absolute value of $\mathrm{CC}$ of "Lena" as host image watermarked with "UT logo".
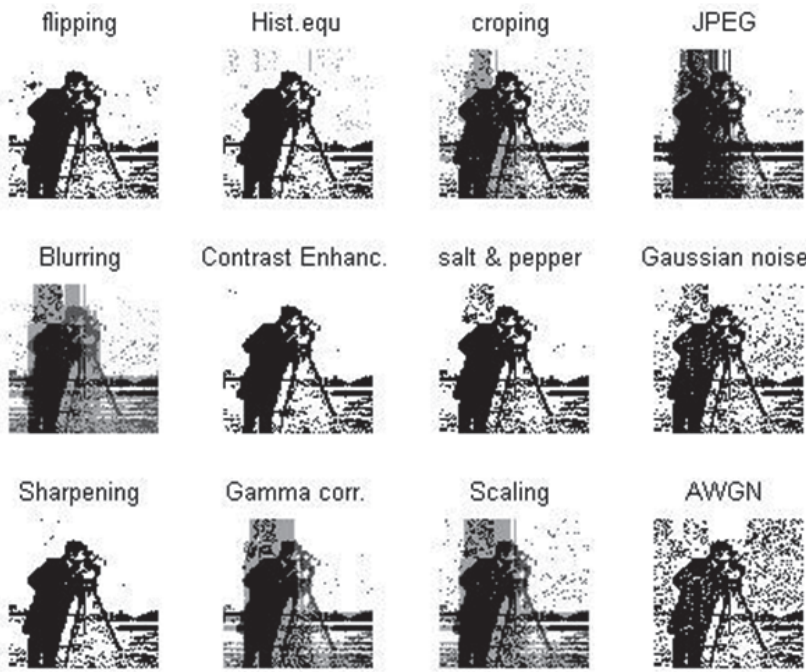

Fig. 5. Extracted black and white watermarks from "Lena" cover image watermarked with "Cameraman".

\section{Conclusion}

This paper presents a new robust watermarking scheme for colored images. It embeds watermark into all three color channel's singular values of the host image. 
First it divides cover image into three RGB color channels and then divides each channel into blocks. Blocks with low entropy value are found and are used for watermark embedding. Each low entropy block is decomposed into frequency channels and further decomposed using CZT. Afterwards orthogonal-triangular decomposition is used to obtain upper-triangular matrix and unitary matrix. After that diagonal matrix is found and singular values are calculated. Watermark image singular values are added with decomposed singular values from original image and watermark is embedded. Experimental results are presented in current work and it is found that the proposed algorithm is robust, imperceptible and it outperforms other state-of-theart watermarking methods.

\section{Acknowledgments}

This work is partially supported by ERDF program "Estonian higher education information and communications technology and research and development activities state program 2011-2015 (ICT program)" and the Estonian Research Council grant PUT (PUT638).

\section{References}

[1] DUTTA, M. K., SINGH, A., ZIA, T. A. An efficient and secure digital image watermarking using features from iris image. In International Conf. on Control Communication and Computing (ICCC). 2013, p. 451-456. DOI: 10.1109/ICCC.2013.6731697

[2] FARFOURA, M. E., HORNG, S.-J. A novel blind reversible method for watermarking relational databases. In International Symposium on Parallel and Distributed Processing with Applications (ISPA). Taipei (Taiwan), 2010, p. 563-569. DOI: 10.1109/ISPA.2010.63

[3] MANJUNATH, M., SIDDAPPAJI, S. A new robust semi blind watermarking using block DCT and SVD. In IEEE International Conference on Advanced Communication Control and Computing Technologies (ICACCCT). 2012, p. 193-197. DOI: 10.1109/ICACCCT.2012.6320769

[4] DORAIRANGASWAMY, M. A., PADHMAVATHI, B. An effective blind watermarking scheme for protecting rightful ownership of digital images. In IEEE Region 10 Conference TENCON. 2009, p. 1 - 6. DOI: 10.1109/TENCON.2009.5395812

[5] KHALIFA, O. O., YUSOF, Y. B., ABDALLA, A.-H., OLANREWAJU, R. F. State-of-the-art digital watermarking attacks. In International Conf. on Computer and Communication Engineering (ICCCE). 2012, p. 744-750. DOI: 10.1109/ICCCE.2012.6271316

[6] RIDZOŃ, R., LEVICKÝ, D. Robust digital watermarking based on the log-polar mapping. Radioengineering, 2007 , vol. 16 , no. 4 , p. 76-81.

[7] NYEEM, H., BOLES, W., BOYD, C. On the robustness and security of digital image watermarking. In International Conference on Informatics, Electronics \& Vision (ICIEV). 2012, p. 1136-1141. DOI: 10.1109/ICIEV.2012.6317496

[8] YUSOF, Y., KHALIFA, O. O. Imperceptibility and robustness analysis of DWT-based digital image watermarking. In International Conference on Computer and Communication Engineering. 2008, p. 1325-1330. DOI: 10.1109/ICCCE.2008.4580820
[9] KORHONEN, J., YOU, J. Peak signal-to-noise ratio revisited: Is simple, is beautiful? In Fourth International Workshop on Quality of Multimedia Experience (QoMEX). 2012, p. 37-38. DOI: 10.1109/QoMEX.2012.6263880

[10] NA, T., KIM, M. A novel no-reference PSNR estimation method with regard to deblocking filtering effect in H.264/AVC bitstreams. IEEE Transactions on Circuits and Systems for Video Technology, 2013, vol. 24, no. 2, p. 320-330. DOI: 10.1109/TCSVT.2013.2255425

[11] NAN, J., JIAN, W., XINXIN, N., YIXIAN, Y. The quantificational relation of imperceptibility, robustness and hiding rate in digital watermarking. In Proceedings of the International Conference on Communications, Circuits and Systems. 2006, vol. 1, p. 11-14. DOI: 10.1109/ICCCAS.2006.284575

[12] XU, W., CHANG, C., HUNG, Y. S., KWAN, S. K., FUNG, P. C. W. Order statistics correlation coefficient as a novel association measurement with applications to biosignal analysis. IEEE Transactions on Signal Processing, 2007, vol. 55, no. 12, p. 5552 to 5563. DOI: 10.1109/TSP.2007.899374

[13] MAHMOOD, A., KHAN, S. Correlation-coefficient-based fast template matching through partial elimination. IEEE Transactions on Image Processing, 2011, vol. 4, no. 21, p. 2099-2108. DOI: 10.1109/TIP.2011.2171696

[14] TAYLOR, R. Interpretation of the correlation coefficient: A basic review. Journal of Diagnostic Medical Sonography, 1990, vol. 6, p. 35-39. DOI: $10.1177 / 875647939000600106$

[15] LARIJANI, H. H., RAD, G. R. A new spatial domain algorithm for gray scale images watermarking. In International Conference on Computer and Communication Engineering, 2008, p. 157-161. DOI: 10.1109/ICCCE.2008.4580587

[16] GHAFOOR, A., IMRAN, M. A non-blind color image watermarking scheme resistant against geometric attacks. Radioengineering, 2012, vol. 21, no. 4, p. 1246-1251.

[17] MEGALINGAM, R. K., NAIR, M. M., SRIKUMAR, R., BALASUBRAMANIAN, V. K. Performance comparison of novel, robust spatial domain digital image watermarking with the conventional frequency domain watermarking techniques. In International Conference on Signal Acquisition and Processing, 2010, p. 349 - 353. DOI: 10.1109/ICSAP.2010.79

[18] LEVICKY, D., FORIS, P. Human visual system models in digital image watermarking. Radioengineering, 2004, vol. 13, no. 4, p. $38-43$.

[19] DEJEY, D., RAJESH, R. S. Robust discrete wavelet-fan beam transforms-based colour image watermarking. IET Image Processing, 2011, vol. 5, no. 4, p. 315-322. DOI: 10.1049/ietipr.2009.0239

[20] YANG, Q., ZHANG, Y., YANG, C., LI, W. Information entropy used in digital watermarking. In Symposium on Photonics and $\begin{array}{llll}\text { Optoelectronics } & \text { (SOPO). } 2012, \quad \text { p. } 1-4 . & \end{array}$ 10.1109/SOPO.2012.6270549

[21] MITRA, P., GUNJAN, R., GAUR, M. S. A multi-resolution watermarking based on contourlet transform using SVD and QR decomposition. In International Conference on Recent Advances in Computing and Software Systems (RACSS), 2012, p. 135-140. DOI: $10.1109 /$ RACSS.2012.6212712

[22] AGOYI, M., ÇELEBI, E., ANBARJAFARI, G. A watermarking algorithm based on chirp z-transform, discrete wavelet transform, and singular value decomposition. Signal, Image and Video Processing, 2014, p. 1-11. DOI: 10.1007/s11760-014-0624-9

[23] GHAZY, R. A., EL-FISHAWY, N. A., HADHOUD, M. M., DESSOUKY, M. I., EL-SAMIE, F. E. A. An efficient block-byblock SVD-based image watermarking scheme. In National Radio Science Conference, NRSC. Cairo (Egypt), 2007, p. 1-9. DOI: 10.1109/NRSC.2007.371376 
[24] SINGH, A. K., SHARMA, N., DAVE, M., MOHAN, A. A novel technique for digital image watermarking in spatial domain. In 2nd IEEE Internat. Conf. on Parallel Distributed and Grid Computing (PDGC), 2012, p. 497-501. DOI: 10.1109/PDGC.2012.6449871

[25] LAI, C.-C., TSAI, C.-C. Digital image watermarking using discrete wavelet transform and singular value decomposition. IEEE Transactions on Instrumentation and Measurement, 2010, vol. 59, no. 11, p. 3060-3063. DOI: 10.1109/TIM.2010.2066770

[26] GHOUTI, L., ANDALUSI, A. M. High-capacity colour image watermarking using multi-dimensional Fourier transforms and semi-random LDPC codes. In IET Conference on Image Processing (IPR 2012). London (UK), 2012, p. 1-5. DOI: $10.1049 / \mathrm{cp} .2012 .0456$

[27] SCHAEFER, G., STICH, M. UCID - An Uncompressed Colour Image Database. Storage and Retrieval Methods and Applications for Multimedia, 2004, p. 472-480. DOI: 10.1117/12.525375

[28] KIM, T.-H., ADELI, H. Advances in Computer Science and Information Technology. Berlin Heidelberg: Springer, 2010. ISSN 0302-9743.

\section{About the Authors ...}

Lauri LAUR received the B. S. degree in Informatics from University of Tartu in 2012. Currently he is an MSc student in Software Engineering of University of Tartu. He is conducting research on watermarking. From 2008 to 2012 he worked as web administrator in local IT Company, IT Grupp AS, and currently he is working as a junior developer in CGI Estonia.
Pejman RASTI received his MSc degree from the Electrical and Electronic Engineering Department, Eastern Mediterranean University in 2014. He has been working in the field of Image Processing and he is currently involved in research projects related to static image and video sharpening, super resolution, and watermarking. He is currently studying as a Ph.D. student in the iCV Group at University of Tartu.

Asst. Prof. Dr. Mary AGOYI is currently a Lecturer at Cyprus International University. She holds Bsc Physics from Benue State University Nigeria and MSc Information Systems Engineering and Ph.D. Computer Engineering from Cyprus International University. Her field of interest includes telecommunication, embedded systems, networking, information security, and image watermarking.

Assoc. Prof. Dr. Gholamreza ANBARJAFARI received his B.Sc., M.Sc., and Ph.D. degrees from the Department of Electrical and Electronic Engineering, Eastern Mediterranean University (EMU), Famagusta, North Cyprus Turkey, in 2007, 2008, 2010 respectively. He has been working in the field of image processing and is currently focusing in many research works related to multimodal emotion recognition, image illumination enhancement, super resolution, image compression, watermarking, visualization and 3D modeling, and computer vision for robotics. He is currently head of iCV Group of IMS Lab and is working as an Assoc. Prof. in the Institute of Technology, University of Tartu, where he established the $\mathrm{iCV}$ research group. 